\section{AB0531 ANTI-CENTROMERE POSITIVE PRIMARY SJÖGREN'S SYNDROME - CHALLENGE TO CLASSIFICATION CRITERIA}

S. Palshina ${ }^{1}$, V. Vasiliev ${ }^{1}$, E. Sokol ${ }^{1}$, B. Chaltsev ${ }^{1}$, E. Rodionova ${ }^{1}$, T. Safonova ${ }^{2} .{ }^{1}$ Nasonova research institute or rheumatology; ${ }^{2}$ Research Institute of Ophthalmic diseases, Moscow, Russian Federation

Background: The prevalence of anti-centromere antibodies (ACA) among patients with primary Sjögren's syndrome (pSS) varies from 3,7\% till 10,9\% [1;2]. They have special course of the disease: 1) with some features of systemic sclerosis (SSc), but rarely evolve to it, 2) higher prevalence of hepatitis and primary biliary cholangitis - PBC.

Objectives: to describe the peculiarities of this subgroup; to evaluate it's conformity to the latest classification criteria for pSS $(2012,2016)[3 ; 4]$ and prevalence of SSc with ACR2013 criteria [5].

Methods: From 2012 to 2016 in a single rheumatic center 50 ACA positive patients (47 female; 3 male) national (Russian) criteria (1987) and in 68\%cases AECG 2002 criteria for pSS (SSc excluded) [Tabl.1]. The mean age was 59,2 $\pm 9,6$ yrs. All diagnostics methods are listed in the table 1, lymphomas were verified pathomorphologicaly with immunohystochemical staining.

Results: In our study 2/3 (64\%) ACA+pts with pSS did't fulfill ACR2012 criteria, because of the lack of aRo or aLa or combination of RF+ANA. According to the criteria, 2016 [4] also just $60-68 \%$ pts will have $\geq 4$ scores for diagnosing pSS. SSc (limited form) due to new criteria [5] might be revealed in $11 / 50(22 \%)$ cases. MALT lymphoma of salivary glands arised in $5 / 6$ pts with visible enlargement of parotid glands.

Table 1. Characteristics of pSS patients with positive ACA

\begin{tabular}{lc}
\hline Parameters & Value $(\mathrm{n}, \%)$ \\
\hline Sialography of parotid gland: sialectasis & $47 / 50(94 \%)$ \\
Xerostomia (grade I-III) stimulated parotid saliva flow rate $<0,5 \mathrm{ml} / 5 \mathrm{~min}$ (III) & $27 / 43(62,8 \%)$ \\
Keratoconjunctivitis sicca with ocular staining score $\geq 5$ & $31 / 50(62 \%)$ \\
Schirmer's test $\leq 5 \mathrm{~mm} / 5$ minutes & $29 / 48(60,4 \%)$ \\
Focal lymphocytic sialadenitis $\geq 1$ foci/4 $\mathrm{mm}^{2}$ & $28 / 30(92 \%)$ \\
Non-Hodgkin lymphoma (incl. MALT lymphoma) & $6(5)=12 \%(10 \%)$ \\
aRo/SS-A $\geq 50 \mathrm{IU} / \mathrm{ml}$ & $16 / 50(32 \%)$ \\
aLa/SS-B $\geq 50 \mathrm{IU} / \mathrm{ml}$ & $3 / 50(6 \%)$ \\
IgM RF positive $\geq 2 \mathrm{UNL}(>30 \mathrm{IU} / \mathrm{ml})$ & $9 / 50(18 \%)$ \\
AMA positive $>10 \mathrm{IU} / \mathrm{ml}$ & $13 / 31(41,9 \%)$ \\
\hline
\end{tabular}

Conclusions: pSS with positive ACA is challenging for diagnostics with comprehensive classification criteria $(32-64 \%$ doesn't meet it [3;4]). These patients overlaps SSc, some PBC. PBC is not rare among, but biliary tract lesions (AMA+(rarely absent, $2 \%$ in our study) with slightly elevated liver enzymes, esp.GGT, and stage 1 typical to PBC without progression) are more common.

\section{References:}

[1] Bournia VK et al. Anticentromere antibody positive Sjögren's Syndrome: a retrospective descriptive analysis. Arthritis Res Ther. 2010;12(2): R47.

[2] Nakamura $\mathrm{H}$ et al. Anti-centromere antibody-seropositive Sjögren's syndrome differs from conventional subgroup in clinical and pathological study. BMC Musculoskelet Disord. 2010 Jul 1;11:140.

[3] Shiboski SC et al. American College of Rheumatology Classification Criteria for Sjögren's Syndrome: A Data-Driven, Expert Consensus Approach in the SICCA Cohort. Arthritis Care Res (Hoboken). 2012; 64(4): 475-487.

[4] Shiboski SC et al. 2016 American College of Rheumatology/European League Against Rheumatism Classification Criteria for Primary Sjögren's Syndrome. ARTHRITIS \& RHEUMATOLOGY. 2016; Vol. 00 (00): p 00-00.

[5] van den Hoogen F et al. 2013 classification criteria for systemic sclerosis: an American College of Rheumatology/European League against Rheumatism collaborative initiative. Arthritis Rheum. 2013 Nov;65(11):2737-47.

Disclosure of Interest: None declared

DOI: 10.1136/annrheumdis-2017-eular.6974

\section{AB0532 VITAMIN D IN A MALAYSIAN LUPUS COHORT AND ITS CORRELATIONS WITH CLINICAL AND IMMUNOLOGICAL PARAMETERS}

S.G. Ong, H.J. Ding. Medicine, Kuala Lumpur Hospital, Kuala Lumpur, Malaysia

Background: Numerous studies have demonstrated that inadequate vitamin $D$ levels are common in systemic lupus erythematosus (SLE) patients and they correlate inversely with disease activity. To date, there is paucity of data on the prevalence of vitamin D status, as well as its clinical and immunological associations in Malaysian SLE patients.

Objectives: Our study aimed to determine the prevalence of vitamin D deficiency and insufficiency in SLE patients, and to examine the associations between vitamin D status with gender, ethnic groups, clinical manifestations of SLE, Schirmer's test, cardiovascular risk factors, bone mineral density and autoantibodies.

Methods: This retrospective study included 216 SLE patients who attended the Rheumatology Clinic of Kuala Lumpur Hospital between January 2013 and December 2015. Data was obtained from patients' medical records. All patients fulfilled the 1997 American College of Rheumatology revised classification criteria for SLE. Serum 25(OH)D concentrations were measured by electrochemiluminescence immunoassay.

Results: A total of 216 SLE patients were included in this study. Eleven (5.1\%) were males and 205 (94.9\%) were females. There were 141 (65.3\%) Malay, 53 (24.5\%) Chinese, $19(8.8 \%)$ Indian and $3(1.4 \%)$ of other ethnic group. This corresponded with the pattern of ethnic distribution in the Malaysian population. Their ages ranged from 14 to 75 years, with a mean age of $35.9 \pm 7.1$ years. Mean duration of SLE at the time of $25(\mathrm{OH}) \mathrm{D}$ analysis was 6.9 years (range from 0 to 39 years), and mean age was $35.1 \pm 6.4$ years (range from 14 to 75 years). Mean $25(\mathrm{OH}) \mathrm{D}$ concentration was $51.3 \pm 14.8 \mathrm{nmol} / \mathrm{L}$ (range from 7.5 to 156.1 $\mathrm{nmol} / \mathrm{L})$. Fifty (23.1\%) patients had vitamin D deficiency, $120(55.6 \%)$ had vitamin D insufficiency, while $46(21.3 \%)$ had adequate vitamin D levels.

Our study showed statistically significant association between vitamin $D$ status and ethnic group $(p<0.001)$. The Chinese ethnic group had the lowest proportion of patients with vitamin D deficiency and insufficiency $(60.4 \%)$, while Malay had the highest proportion at $86.5 \%$. Mean levels of serum $25(\mathrm{OH}) \mathrm{D}$ in Chinese, Indian and Malay SLE patients were $66.3 \pm 36.7 \mathrm{nmol} / \mathrm{L}, 54.9 \pm 36.4 \mathrm{nmol} / \mathrm{L}$, and $45.0 \pm 27.5 \mathrm{nmol} / \mathrm{L}$, respectively. Among the clinical manifestations of SLE, only lupus nephritis showed a statistically significant association with vitamin $D$ status $(p<0.001)$. In terms of cardiovascular risk factors, hypertension demonstrated significant correlation with vitamin $D$ status $(p=0.032)$.

No significant association was found between vitamin $D$ status and gender. Nonetheless, male SLE patients had higher mean $25(\mathrm{OH}) \mathrm{D}$ concentrations at $69.4 \pm 36.9 \mathrm{nmol} / \mathrm{L}$, compared to female at $50.2 \pm 31.6 \mathrm{nmol} / \mathrm{L}$. There were no significant correlations between vitamin D status and clinical features of lupus such as cutaneous lesions, arthritis and NPSLE; positive Schirmer's test; cardiovascular risk factors, that are, dyslipidemia and diabetes mellitus; osteoporosis; and autoantibodies which included anti-dsDNA antibody, anti-Ro antibody, anticardiolipin antibody and rheumatoid factor.

Conclusions: Sub-optimal vitamin D levels are prevalent among SLE patients in a tropical country and are associated with ethnic group, lupus nephritis and hypertension. It is essential to include vitamin D supplementation in the management of SLE patients.

References:

[1] Toloza SMA, Cole DEC, Gladman DD, et al. Vitamin D insufficiency in a large female SLE cohort. Lupus 2010;19: 13-19.

Disclosure of Interest: None declared

DOI: 10.1136/annrheumdis-2017-eular.1100

\section{AB0533 JUVENILE AND JUVENILE-ONSET SYSTEMIC LUPUS ERYTHEMATOSUS PATIENTS: CLINICAL CHARACTERISTICS, DISEASE ACTIVITY AND DAMAGE}

T.A. Gheita ${ }^{1}$, R.E. Gheith ${ }^{1}$, H.S. El-Fishawy ${ }^{2}$, D.M.R. Bahgat ${ }^{3}$, A.M. Nour El-Din ${ }^{4} .{ }^{1}$ Rheumatology and Clinical Immunology; ${ }^{2}$ Internal Medicine; ${ }^{3}$ Clinical and Chemical Pathology, Faculty of Medicine - Kasr Al-Ainy School of Medicine Cairo University, Cairo; ${ }^{4}$ Pediatrics, National Research Centre (NRC), Giza, Egypt

Background: The diagnosis of systemic lupus erythematosus (SLE) in children is challenging as the heterogeneous manifestations and disease impact on the child's growth highlighted the importance of timely diagnosis and management.

Objectives: The aim of the present study was to assess and compare the clinical characteristics, disease activity and damage between juvenile (JSLE) and juvenile-onset (JO-SLE) Egyptian patients.

Methods: Seventy-eight SLE patients (26 JSLE and 52 JO-SLE) were included in this study. Disease activity was assessed using the SLE Disease Activity Index (SLEDAI) and organ damage using the Systemic Lupus International Collaborating Clinics (SLICC) index.

Results: The mean age of the JSLE children was $13.25 \pm 2.09$ years and $23.17 \pm 4.26$ years for JO-SLE cases. JO-SLE cases were older at disease onset with a higher female-to-male ratio. There were no noticeable gender differences. There was a significantly higher frequency of serositis, nephritis and hematological involvement in the JO-SLE $(57.7 \%, 76.9 \%$ and $73.1 \%)$ compared to the JSLE cases $(15.4 \% ; 30.8 \%$ and $30.8 \%)(p<0.001$ for all). The erythrocyte sedimentation rate, creatinine and proteinuria were significantly increased in JO-SLE while alkaline phosphatase was higher in JSLE cases. In JO-SLE cases, SLEDAI significantly increased $(5.96 \pm 6.18$ vs $3.12 \pm 1.97 ; p=0.003)$ and the SLICC tended to increase compared to the JSLE children. More JO-SLE cases received hydroxychloroquine and azathioprine.

Conclusions: The existence of differences in clinical phenotype has been confirmed, between JSLE and JO-SLE especially as regards serositis, nephritis and heamatological affection. The disease damage was comparable which denotes that the maximum organ involvement occurs in childhood with an almost stationary course. Rheumatologists caring for children with SLE must be aware of the greater risk of major haematological and renal involvement as well as important long-term morbidity.

Disclosure of Interest: None declared

DOI: 10.1136/annrheumdis-2017-eular.2993 